\title{
Discrete Random Walks on One-Sided "Periodic" Graphs
}

\author{
Michael Drmota
}

Department of Geometry, TU Wien, Wiedner Hauptstrasse 8-10, A-1040 Vienna, Austria

michael.drmota@tuwien.ac.at

In this paper we consider discrete random walks on infinite graphs that are generated by copying and shifting one finite (strongly connected) graph into one direction and connecting successive copies always in the same way. With help of generating functions it is shown that there are only three types for the asymptotic behaviour of the random walk. It either converges to the stationary distribution or it can be approximated in terms of a reflected Brownian motion or by a Brownian motion. In terms of Markov chains these cases correspond to positive recurrence, to null recurrence, and to non recurrence.

Keywords: discrete random walk, generating functions, singularity analysis

\section{Introduction}

The purpose of this paper is to consider random walks on infi nite graphs $G$ of the following type. Let $K$ and $L$ be a fi nite strongly connected di-graphs and $K_{0}, K_{1}, K_{2}, \ldots$ copies of $K$. The set of nodes, $V(G)$, of $G$ is now given by $V(L) \cup V\left(K_{0}\right) \cup V\left(K_{1}\right) \cup \cdots$. The directed edges of $G, E(G)$, consist fi rst of the edges $E(L) \cup E\left(K_{0}\right) \cup E\left(K_{1}\right) \cup \cdots$ and second of edges between $L$ and $K_{0}$, between $K_{0}$ and $K_{1}$, between $K_{1}$ and $K_{2}$ etc., where the edges from $K_{j}$ to $K_{j+1}$ are the same for all $j=0,1,2, \ldots$. We also assume that every node of $K_{0}$ has the same outdegree as $K_{j}$ for $j=1,2, \ldots$, that is, every directed edge from $K_{j+1}$ to $K_{j}$ (for $j=0,1,2, \ldots)$ has a counterpart from $K_{0}$ to $L$.

We consider a discrete random walk $X_{n}$ (as a Markov chain) on $G$, where the starting point $X_{0}$ is in $L$. We also assume that the transition probabilities of the corresponding nodes of $K_{j}$ are the same for all $j=0,1,2, \ldots$.

The simplest case is the one-sided "linear" graph, where $L$ and $K_{j}$ have size 1 (see Figure 1). Of course, the corresponding random walk is just a Markov chain on the non-negative integers with reflection at zero, see Feller $(1968,1971)$, or a discrete time version of the continuous time Markov chain modeling a M/M/1 queue (see Neuts $(1981,1989)$; Latouche and Ramaswami (1999)). It is well known that the random walk $X_{n}$ on $G$ is either positive recurrent, null recurrent, or non recurrent.

The general case corresponds to a discrete time version of a homogeneous quasi-birth-and-death process (see Neuts $(1981,1989)$; Latouche and Ramaswami (1999)) that is characterised by a Neuts structure given 


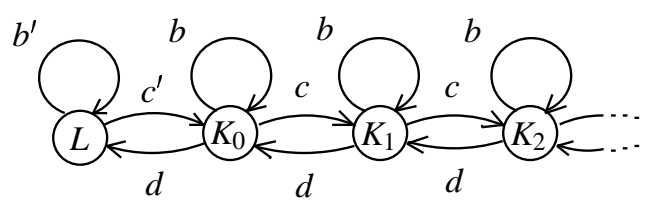

Fig. 1: One-sided "linear" graph (with transition probabilities)

by an infi nite matrix of the form

$$
\left(\begin{array}{cccccc}
\mathbf{B}^{\prime} & \mathbf{C}^{\prime} & \mathbf{0} & \mathbf{0} & \ldots & \\
\mathbf{D}^{\prime} & \mathbf{B} & \mathbf{C} & \mathbf{0} & \ldots & \\
\mathbf{0} & \mathbf{D} & \mathbf{B} & \mathbf{C} & \mathbf{0} & \ldots \\
\mathbf{0} & \mathbf{0} & \mathbf{D} & \mathbf{B} & \mathbf{C} & \ldots \\
& & \ddots & \ddots & \ddots & \ddots
\end{array}\right)
$$

where the (fi nite) matrices $\mathbf{B}, \mathbf{C}^{\prime}, \mathbf{D}^{\prime}, \mathbf{B}, \mathbf{C}, \mathbf{D}$ collect the transition probabilities (see Section 3 ). These kinds of graphs also appear in performance evaluation, for example, compare with Hermanns et al. (2002).

It is also worth mentioning that there are specifi c problems in combinatorics, where graphs of this type appear, for example, the graph presented in Figure $2^{\dagger}$ is related to a problem of bin-packing (see Prodinger $(1985,1990))$.

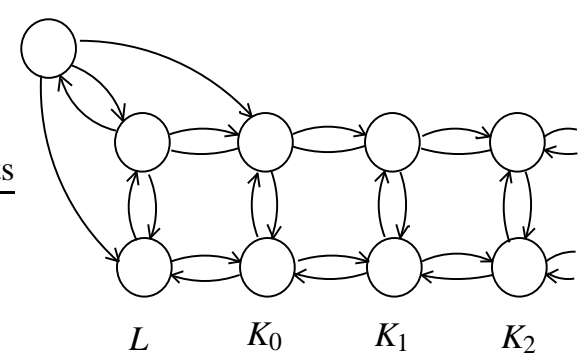

Fig. 2: Graph related to bin-packing

The main purpose of this paper is to indicate that depending on the transition probabilities there are three typical asymptotic behaviours of $X_{n}$. It either converges to the stationary distribution or it can be approximated in terms of a reflected Brownian motion or by a Brownian motion. ${ }^{\ddagger}$ In terms of Markov chains these cases correspond to positive recurrence, to null recurrence, and to non recurrence.

\footnotetext{
$\dagger$ This example was in fact the motivating example for writing this paper.

\# We only present one dimensional distributional results. However, with help of the same proof techniques we easily obtain corresponding functional versions. One has to show fi nite-dimensional distributional results and tightness. Both properties can be shown with help of analytic methods applied to corresponding multivariate generating functions, for related problems and methods see Drmota and Gittenberger (1997) or Drmota et al. (2001).
} 
In what follows we present a unifi ed approach to these kinds of problems that is bases on generating functions and on analytic methods (singularity analysis, saddle point techniques) for obtaining asymptotic relations for the coeffi cients via Cauchy's formula. It seems that this kind of method has not been used in this context in this generality.

It also seems that the precise statements given below (in particular the second and third part of Theorem 2) are new in this generality. The case of positive recurrence (of Theorem 2) has been discussed in detail (see Latouche and Ramaswami (1999)). Also, it is well known that Dyck paths and Motzkin paths can be approximated by a reflected Brownian motion. Further, the paper of Lalley (2001) deals with random walks on regular languages - it seems that our case may be viewed as special cases - but the results there concern only asymptotic expansions for the probabilities $\operatorname{Pr}\left\{X_{0}=v, X_{n}=w\right\}$, where $v, w \in V(G)$ are fi xed and $n$ tends to infi nity, compare also with Lalley (1995).

It would be interesting, too, to extend the present results to graphs $G$ with specifi c infi nite graphs $L$ and $K_{j}$. This would cover one-sided versions of the random walk on the $d$-dimensional grid. (One can either try to use the method by Lalley (2002) for infi nite systems of functional equations or the Fourier analytic methods by Guivarc'h (1984) and Krámli and Szász (1984), compare also with (Woess, 2000, Section 13).)

In section 2 we fi rst consider the simplest case of a one-sided "linear" graph (see Figure 1) that is related to the classical random walk on the non-negative integers (Dyck paths, Motzkin paths etc.). The general case will then treated in section 3 .

\section{The one-sided "linear" graph}

\subsection{Statement of the Result}

In this section we will describe in detail the asymptotic behaviour of $X_{n}$ with $X_{0}=L$ for the one-sided "linear" graph $G$, where $b, c, d$ and $b^{\prime}, c^{\prime}$ denote the corresponding transition probabilities (compare with Figure 1). ${ }^{\S}$

Theorem 1. Suppose that $b, c, d$ and $b^{\prime}, c^{\prime}$ are positive numbers with $b+c+d=b^{\prime}+c^{\prime}=1$; and let $X_{n}$ be the random walk on the one-sided "linear" graph $G$ with $X_{0}=L$.

1. If $c<d$ then we have

$$
\lim _{n \rightarrow \infty} \operatorname{Pr}\left\{X_{n}=L\right\}=\frac{d-c}{d-c+c^{\prime}} \quad \text { and } \quad \lim _{n \rightarrow \infty} \operatorname{Pr}\left\{X_{n}=K_{\ell}\right\}=\frac{c^{\prime}(d-c)}{d\left(d-c+c^{\prime}\right)}\left(\frac{c}{d}\right)^{\ell} \quad(\ell \geq 0) .
$$

that is, $X_{n}$ is positive recurrent. The distribution of $X_{n}$ converges to the stationary distribution.

2. If $c=d$ then $X_{n} / \sqrt{c n}$ is null recurrent and converges weakly to the absolute normal distribution. In particular, we have, as $n \rightarrow \infty$,

$$
\operatorname{Pr}\left\{X_{n}=L\right\}=\frac{1}{c^{\prime}} \sqrt{\frac{2 c}{n \pi}}+O\left(\frac{1}{n}\right)
$$

\footnotetext{
$\S$ Theorem 1 is surely not new, but it seems that the proof method is. Furthermore, it plays the rôle of a prototype for the general case covered by Theorem 2 .
} 
and (uniformly for all $\ell \geq 0$ )

$$
\operatorname{Pr}\left\{X_{n}=K_{\ell}\right\}=\sqrt{\frac{2}{n c \pi}} \exp \left(-\frac{\ell^{2}}{2 c n}\right)+O\left(\frac{1}{n}\right) .
$$

3. If $c>d$ then $X_{n}$ is non recurrent and $\left(X_{n}-(c-d) n\right) / \sqrt{\left(c+d-(c-d)^{2}\right) n}$ converges weakly to the standard normal distribution. We also have, as $n \rightarrow \infty$ and uniformly for all $\ell \geq 0$,

$$
\operatorname{Pr}\left\{X_{n}=K_{\ell}\right\}=\frac{1}{\sqrt{2 \pi\left(c+d-(c-d)^{2}\right) n}} \exp \left(-\frac{(\ell-(c-d) n)^{2}}{2\left(c+d-(c-d)^{2}\right) n}\right)+O\left(\frac{1}{n}\right) .
$$

Note that the assumption that $b>0$ and $b^{\prime}>0$ are not that restrictive. In particular if one of them is zero then the result remains true as it is. Only if both are zero then $X_{n} \notin K_{\ell}$ (and consequently $\operatorname{Pr}\left\{X_{n} \in K_{\ell}\right\}=0$ ) if $n$ and $\ell$ have the same parity. However, if $n \not \equiv \ell \bmod 2$ then we get qualitatively the same result (and the proofs are a little bit more technical).

Note also that the probabilities $\operatorname{Pr}\left\{X_{n}=L\right\}$ have been discussed in Lalley (2001) for the case $b=0$ and $b^{\prime}>0$.

Finally, as mentioned above, with a little bit more effort it can be shown that in the case $c=d$ the normalized discrete processes

$$
\left(\frac{X_{\lfloor t n\rfloor}}{\sqrt{c n}}, t \geq 0\right)_{n \geq 1}
$$

converges weakly to a reflected Brownian motion as $n \rightarrow \infty$; and for $c<d$ the processes

$$
\left(\frac{X_{\lfloor t n\rfloor}-t(c-d) n}{\sqrt{\left(c+d-(c-d)^{2}\right) n}}, t \geq 0\right)_{n \geq 1}
$$

converges weakly to the standard Brownian motion.

\subsection{Generating Functions}

We start with a property of one-sided paths on the integers (see Figure 3).

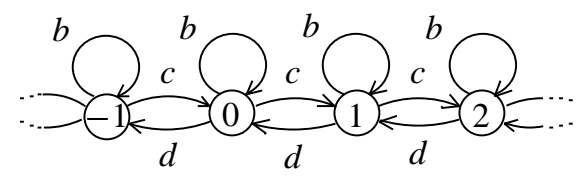

Fig. 3: Random walk on the integers

Lemma 1. Let $Y_{n}$ denote the random walk on the integers (see Figure 3) with $Y_{0}=0$. Then the generating function of one-sided return probabilities

$$
M(x)=\sum_{n \geq 0} \operatorname{Pr}\left\{Y_{1} \geq 0, Y_{2} \geq 0, \ldots, Y_{n-1} \geq 0, Y_{n}=0\right\} \cdot x^{n}
$$


satisfies the functional equation

$$
M(x)=1+b x M(x)+c d x^{2} M(x)^{2}
$$

and is thus explicitly given by

$$
M(x)=\frac{1-b x-\sqrt{(1-b x)^{2}-4 c d x^{2}}}{2 c d x^{2}} .
$$

The radius of convergence $x_{0}$ is given by

$$
x_{0}=\frac{1}{b+2 \sqrt{c d}}=\frac{1}{1-(\sqrt{c}-\sqrt{d})^{2}} .
$$

If $b>0$ then $x_{0}$ is also the only singularity on the circle of convergence $|x|=x_{0}$. Furthermore, $M(x)$ has a local expansion of the form

$$
M(x)=\frac{b+2 \sqrt{c d}}{\sqrt{c d}}-\frac{1}{\sqrt{2}}\left(\frac{b+2 \sqrt{c d}}{\sqrt{c d}}\right)^{3 / 2} \cdot \sqrt{1-(b+2 \sqrt{c d}) x}+O(1-(b+2 \sqrt{c d}) x)
$$

around its singularity $x=x_{0}$.

Note that the generating function $M(x)$ is closely related to the generating functions $U(x), G(x)$, and $R(x)$ presented in (Latouche and Ramaswami, 1999, p. 96). We have $M(x)=1 /(1-U(x)), G(x)=$ $M(x) \cdot d x$, and $R(x)=c x \cdot M(x)$.

Proof. The functional equation (2) is immediately clear by writing it in the following way:

$$
M(x)=1+b x \cdot M(x)+c x \cdot M(x) \cdot d x \cdot M(x) .
$$

If the fi rst step is the loop (with probability $b$ ) then the remaining part is just a non-negative path from 0 to 0 and the contribution is $b x \cdot M(x)$. If the fi rst step goes to the right (with probability $c$ ) then we decompose the path into four parts: fi rst the step from 0 to the right, then we consider the part from 1 to 1 that is followed by the fi rst step back from 1 to 0 , the third part is this step back, and the last part is again a non-negative path from 0 to 0 . Hence, in terms of generating functions this case contributed $c x \cdot M(x) \cdot d x \cdot M(x)$. This proves (2).

The remaining properties follow directly from (2).

Next consider the original one-sided "linear" graph.

Lemma 2. Let $X_{n}$ denote the random walk on the graph represented by Figure 1 and set

$$
M_{L}(x)=\sum_{n \geq 0} \operatorname{Pr}\left\{X_{n}=L\right\} \cdot x^{n} \quad \text { and } \quad M_{\ell}(x)=\sum_{n \geq 0} \operatorname{Pr}\left\{X_{n}=K_{\ell}\right\} \cdot x^{n} \quad(\ell \geq 0) .
$$

Then

$$
M_{L}(x)=\frac{1}{1-b^{\prime} x-c^{\prime} d x^{2} M(x)} \quad \text { and } \quad M_{\ell}(x)=\frac{c^{\prime}}{c} \frac{(c x M(x))^{\ell+1}}{1-b^{\prime} x-c^{\prime} d x^{2} M(x)} \quad(\ell \geq 0)
$$


Proof. With help of the same reasoning as in the proof of Lemma 1 one gets the relation

$$
M_{L}(x)=1+b^{\prime} x M_{L}(x)+c^{\prime} x M(x) d x M_{L}(x)
$$

that proves the proposed representation for $M_{L}(x)$.

Next we have $M_{0}(x)=M_{L}(x) c^{\prime} x M(x)$. Here we have to divide all paths from $L$ to $K_{0}$ into three parts. The first part is just the path from $L$ to $L$ that is followed by the last step from $L$ to $K_{0}$. This step is the second part, and the third part is a non-negative path from $K_{0}$ to $K_{0}$. In a similar way we also obtain the recurrence $M_{\ell+1}(x)=M_{\ell}(x) \operatorname{cx} M(x)$. This completes the proof of Lemma 2 .

\subsection{Analytic Methods}

We now use the above explicit representations for $M_{L}(x)$ and $M_{\ell}(x)(\ell \geq 0)$ and Cauchy's formula to extract the coeffi cients, e.g.

$$
\operatorname{Pr}\left\{X_{n}=K_{\ell}\right\}=\frac{1}{2 \pi i} \int_{|x|=r} \frac{M_{\ell}(x)}{x^{n+1}} d x,
$$

where $r$ is smaller that the radius of convergence of $M_{\ell}(x)$. By shifting the path of integration suitably in the analyticity region of $M_{\ell}(x)$ and evaluating asymptotically the integral we will thus obtain asymptotic expansions for $\operatorname{Pr}\left\{X_{n}=K_{\ell}\right\}$. In particular we have to deal with three different cases, fi rst with a polar singularity, second with a square-root singularity, and third we have to apply saddle point techniques. These kinds of techniques are very well established in the literature. Therefore we will not work out all the details but refer to proper references (e.g. to Drmota (1994)).

We start with the case $c<d$.

Lemma 3. Suppose that $c<d$. Then the radius of convergence of $M_{L}(x)$ and $M_{\ell}(x)(\ell \geq 0)$ is $x_{1}=1$ that is also a polar singularity of order 1 . Furthermore, we have

$$
\lim _{n \rightarrow \infty} \operatorname{Pr}\left\{X_{n}=L\right\}=\frac{d-c}{d-c+c^{\prime}} \quad \text { and } \quad \lim _{n \rightarrow \infty} \operatorname{Pr}\left\{X_{n}=K_{\ell}\right\}=\frac{c^{\prime}(d-c)}{d\left(d-c+c^{\prime}\right)}\left(\frac{c}{d}\right)^{\ell} \quad(\ell \geq 0) .
$$

Proof. First note that (for $c<d$ ) we have $M(1)=1 / d$ and $M^{\prime}(1)=(1-d+c) /(d(d-c))$. Thus,

$$
1-b^{\prime} x-c^{\prime} d x^{2} M(x)=\frac{d-c+c^{\prime}}{d-c}(1-x)+O\left((1-x)^{2}\right) .
$$

and consequently

$$
M_{L}(x)=\frac{d-c}{d-c+c^{\prime}} \frac{1}{1-x}+\text { analytic function }
$$

and

$$
M_{\ell}(x)=\frac{c^{\prime}(d-c)}{d\left(d-c+c^{\prime}\right)}\left(\frac{c}{d}\right)^{\ell} \frac{1}{1-x}+\text { analytic function }
$$

for $|x|<1 /(b+2 \sqrt{c d})$. (Note that $1 /(b+2 \sqrt{c d})>1$.) Of course, this directly implies (4).

The most interesting case is the case $c=d$. 
Lemma 4. Suppose that $c=d$. Then the radius of convergence of $M_{L}(x)$ and $M_{\ell}(x)(\ell \geq 0)$ is $x_{1}=1$ that is an algebraic singularity. Here we get, as $n \rightarrow \infty$,

$$
\operatorname{Pr}\left\{X_{n}=L\right\}=\frac{1}{c^{\prime}} \sqrt{\frac{2 c}{n \pi}}+O\left(\frac{1}{n}\right) .
$$

and (uniformly for all $\ell \geq 0$ )

$$
\operatorname{Pr}\left\{X_{n}=K_{\ell}\right\}=\sqrt{\frac{2}{n c \pi}} \exp \left(-\frac{\ell^{2}}{2 c n}\right)+O\left(\frac{1}{n}\right) .
$$

Proof. The essential difference between the present case and that of Lemma 3 is that $M(x)$ is not regular at $x=1$. We have to use the singular expansion (3) of Lemma 1 and obtain (around $x=1$ )

$$
1-b^{\prime} x-c^{\prime} d x^{2} M(x)=\frac{c^{\prime}}{\sqrt{2 c}} \sqrt{1-x}+O(1-x) .
$$

Furthermore

$$
(\operatorname{cxM}(x))^{\ell}=\exp \left(-\frac{\ell}{\sqrt{2 c}} \sqrt{1-x}+O(\ell(1-x))\right) .
$$

Thus, the dominant behaviour of $M_{\ell}(x)$ around $x_{0}=1$ is of the form

$$
\sqrt{\frac{2}{c}} \cdot \frac{\exp \left(-\frac{\ell}{\sqrt{2 c}} \sqrt{1-x}\right)}{\sqrt{1-x}}
$$

We can now proceed as in the proof of Theorem 4 of Drmota (1994). We just have to use the formula

$$
\frac{1}{2 \pi i} \int_{\gamma} \frac{e^{-\lambda \sqrt{-t}-t}}{\sqrt{-t}} d t=\frac{1}{\sqrt{\pi}} e^{-\lambda^{2}}
$$

where $\gamma$ denotes a Hankel contour. This directly leads to (5) and (6).

The analysis of the fi nal case $c>d$ is a little bit different from the previous ones. In the fi rst two cases the singular behaviour of $M_{L}(x)$ and $M_{\ell}(x)$ around the point $x_{0}=1$ has governed the asymptotic behaviour of the coeffi cients. In the third case we will again work around the critical point $x_{0}=1$ but now with help of a saddle point method. The radius of convergence is larger than 1.

Lemma 5. Suppose that $c>d$. Then $X_{n}$ satisfies a central limit theorem with mean value $\mathbf{E} X_{n} \sim(c-d) n$ and $\operatorname{Var} X_{n} \sim\left(c+d-(c-d)^{2}\right) n$. In particular we have the following local limit theorem as $n \rightarrow \infty$ and uniformly for all $\ell \geq 0$ :

$$
\operatorname{Pr}\left\{X_{n}=K_{\ell}\right\}=\frac{1}{\sqrt{2 \pi\left(c+d-(c-d)^{2}\right) n}} \exp \left(-\frac{(\ell-(c-d) n)^{2}}{2\left(c+d-(c-d)^{2}\right) n}\right)+O\left(\frac{1}{n}\right) .
$$

Proof. Note fi rst that $M_{\ell}(x)=\frac{c^{\prime}}{c} M_{L}(x)(c x M(x))^{\ell+1}$ and that $x_{1}=1$ is a regular point of $M_{\ell}(x)$. Thus, $M_{\ell}(x)$ is (despite of an analytic factor) a power of the function $\operatorname{cxM}(x)$. Consequently, we can directly apply the (saddle point) methods of Drmota (1994) and obtain the result. 


\section{The general case}

\subsection{Matrices of Generating Functions}

We are now going to consider the general situation. We will denote by $\mathbf{B}, \mathbf{C}, \mathbf{D}$ the corresponding matrices containing the transition probabilities inside $K_{j}$, from $K_{j}$ to $K_{j+1}$, from $K_{j+1}$ to $K_{j}$ and by and $\mathbf{B}^{\prime}, \mathbf{C}^{\prime}, \mathbf{D}^{\prime}$ the transition probabilities inside $L$, from $L$ to $K_{0}$ and from $K_{0}$ to $L$. (Note that in contrast to the "linear" case $\mathbf{D}$ and $\mathbf{D}^{\prime}$ are different in general.)

We now assume that the random walk $X_{n}$ starts at a vertex $w$ in $L$.

The fi rst (and easy) step is to generalize the above relations for generating functions. Let $\mathbf{M}_{L}(x)=$ $\left(M_{L ; w, w^{\prime}}(x)\right)_{w, w^{\prime} \in V(L)}$ denote the matrix of the generating functions

$$
M_{L ; w, w^{\prime}}(x)=\sum_{n \geq 0} \operatorname{Pr}\left\{X_{0}=w, X_{n}=w^{\prime}\right\} \cdot x^{n}
$$

and $\mathbf{M}_{\ell}(x)=\left(M_{\ell ; w, v}(x)\right)_{w \in V(L), v \in K_{\ell}}$ the matrix of functions

$$
M_{\ell ; w, v}(x)=\sum_{n \geq 0} \operatorname{Pr}\left\{X_{0}=w, X_{n}=v\right\} \cdot x^{n} .
$$

Lemma 6. Let $\mathbf{M}(x)=\left(M_{v, v^{\prime}}(x)\right)_{v, v^{\prime} \in K}$ denote the (analytic) solution with $\mathbf{M}(0)=\mathbf{I}$ of the matrix equation

$$
\mathbf{M}(x)=\mathbf{I}+x \mathbf{B} \mathbf{M}(x)+x^{2} \mathbf{C M}(x) \mathbf{D} \mathbf{M}(x) .
$$

Then $\mathbf{M}_{L}(x)$ and $\mathbf{M}_{\ell}(x)$ are given by

$$
\mathbf{M}_{L}(x)=\left(\mathbf{I}-x \mathbf{B}^{\prime}-x^{2} \mathbf{C}^{\prime} \mathbf{M}(x) \mathbf{D}^{\prime}\right)^{-1}
$$

and (for $\ell \geq 0)$

$$
\mathbf{M}_{\ell}(x)=x^{\ell+1}\left(\mathbf{I}-x \mathbf{B}^{\prime}-x^{2} \mathbf{C}^{\prime} \mathbf{M}(x) \mathbf{D}^{\prime}\right)^{-1} \mathbf{C}^{\prime} \mathbf{M}(x)(\mathbf{C M}(x))^{\ell} .
$$

Proof. The proof is exactly the same as that of Lemma 1 and 2 and already appears (for the case of Figure 2) in Prodinger (1990), compare also with Kuich and Urbanek (1983). For $x=1$ the matrix $\mathbf{M}=\mathbf{M}(1)$ is also related to the matrices $\mathbf{U}, \mathbf{G}$, and $\mathbf{R}$ of (Latouche and Ramaswami, 1999, p. 137), in particular, $\mathbf{M}=(\mathbf{I}-\mathbf{U})^{-1}, \mathbf{G}=\mathbf{M D}$, and $\mathbf{R}=\mathbf{C M}$.

The main difference to the "linear" case is that we are now not able to solve the above system (8)-(10) explicitly. Nevertheless, from an analytic point of view they behave in a similar way. Let us start with $\mathbf{M}(x)$, the solution of (8).

Lemma 7. Suppose that $\mathbf{B}$ is a primitive irreducible matrix and let $\mathbf{M}(x)=\left(M_{v, v^{\prime}}(x)\right)_{v, v^{\prime} \in V(K)}$ denote the solution of (8). Then all functions $M_{v, v^{\prime}}(x)$ have a common radius of convergence $x_{0} \geq 1$. Furthermore, $x_{0}$ is the only singularity on the circle of convergence $|x|=x_{0}$ and there is a local expansion of the form

$$
\mathbf{M}(x)=\tilde{\mathbf{M}}_{1}-\tilde{\mathbf{M}}_{2} \sqrt{1-\frac{x}{x_{0}}}+O\left(1-\frac{x}{x_{0}}\right)
$$

around its singularity $x=x_{0}$, where $\tilde{\mathbf{M}}_{1}$ and $\tilde{\mathbf{M}}_{2}$ are matrices with positive elements. 
Proof. The relation (8) is a system of $|V(K)|^{2}$ algebraic equation for the functions $M_{v, v^{\prime}}(x)$ that can be written in the form $\mathbf{Q}(x)=\mathbf{F}(x, \mathbf{Q}(x))$, where $\mathbf{Q}(x)$ is just the vector of functions $M_{w, w^{\prime}}(x)$ and $\mathbf{F}(x, \mathbf{y})$ is a proper (non-linear) polynomial vector function with non-negative coeffi cients. By assumption $\mathbf{B}$ is irreducible (and non-negative). Thus, the so-called dependency graph (compare with Drmota (1997)) of this system is strongly connected, that is, it is impossible to solve a subsystem before solving the whole system. Consequently, all (algebraic) functions $M_{v, v^{\prime}}(x)$ have the same fi nite radius of convergence and by Lalley (2001) (compare also with Drmota (1997)) they have a square-root singularity at $x=x_{0}$ of the form (11), where all entries of $\tilde{\mathbf{M}}_{1}$ and $\tilde{\mathbf{M}}_{2}$ are positive.

The assumption that $\mathbf{B}$ is primitive implies that all (suffi ciently large) coeffi cients of the power series $M_{v, v^{\prime}}(x)$ are positive. This property shows that $x=x_{0}$ is the only singularity on the circle of convergence $|x|=x_{0}$ (compare with Drmota (1997), where this property is called of simple type).

Finally, we surely have $x_{0} \geq 1$. For, if $x_{0}<1$ then the coeffi cients of $M_{v, v^{\prime}}(x)$ are unbounded. However, the coeffi cients of $M_{v, v^{\prime}}(x)$ are probabilities (compare also with (1)) and thus bounded. This completes the proof of the lemma.

This lemma also shows that all entries of the matrix function

$$
\mathbf{M}_{L}(x)=\left(\mathbf{I}-x \mathbf{B}^{\prime}-x^{2} \mathbf{C}^{\prime} \mathbf{M}(x) \mathbf{D}^{\prime}\right)^{-1}
$$

have a fi nite radius of convergence $x_{1}$ that satisfi es

$$
1 \leq x_{1} \leq x_{0}
$$

(Note that $x_{1}$ cannot be smaller than 1 since the coeffi cients are probabilities and thus bounded.)

\subsection{A General Theorem}

As in the "linear" case there are three kinds of asymptotic behaviours for $X_{n}$, where we assume that $X_{0}=w_{0}$ with a given node $w_{0} \in V(L)$.

Theorem 2. Suppose that the matrices $\mathbf{B}$ and $\mathbf{B}^{\prime}$ are primitive irreducible, that no row of $\mathbf{C}$ is zero, and that the matrices $\mathbf{D}, \mathbf{C}^{\prime}, \mathbf{D}^{\prime}$ are non-zero. Let $X_{n}$ denote the random walk on $G$ with $X_{0}=w_{0} \in V(L)$ and let $x_{0}$ and $x_{1}$ denote the radius of convergence of the entries of $\mathbf{M}(x)$ and $\mathbf{M}_{L}(x)$.

1. If $x_{0}>1$ and $x_{1}=1$ then $X_{n}$ is positive recurrent and for all $v \in V(G)=V(L) \cup V\left(K_{0}\right) \cup V\left(K_{1}\right) \cup \cdots$ we have

$$
\lim _{n \rightarrow \infty} \operatorname{Pr}\left\{X_{n}=v\right\}=p_{v}
$$

where $\left(p_{v}\right)_{v \in V(G)}$ is the (unique) stationary distribution on G. Furthermore, there exists a nonnegative matrix $\mathbf{R}$ (where all eigenvalues have moduli $<1$ ) such that

$$
\mathbf{p}_{\ell+1}=\mathbf{p}_{\ell} \mathbf{R}
$$

in which $\mathbf{p}_{\ell}=\left(p_{v}\right)_{v \in K_{\ell}}$.

2. If $x_{0}=x_{1}=1$ then $X_{n}$ is null recurrent and there exist $\rho_{v^{\prime}}>0\left(v^{\prime} \in V(K)\right), \rho_{w}^{\prime}>0(w \in V(L))$ and $\eta>0$ such that, as $n \rightarrow \infty$,

$$
\operatorname{Pr}\left\{X_{n}=w\right\}=\rho_{w}^{\prime} \sqrt{\frac{1}{n \pi}}+O\left(\frac{1}{n}\right) \quad(w \in V(L)) .
$$


and (uniformly for all $\ell \geq 0$ )

$$
\operatorname{Pr}\left\{X_{n}=v\right\}=\rho \sim \sqrt{\frac{1}{n \pi}} \exp \left(-\frac{\ell^{2}}{2 \eta n}\right)+O\left(\frac{1}{n}\right) \quad\left(v \in V\left(K_{\ell}\right)\right),
$$

where $\tilde{v}\left(\right.$ for $\left.v \in K_{\ell}\right)$ denotes the corresponding node in $K$.

3. If $x_{1}>1$ then $X_{n}$ is non recurrent and there exist $\tau_{v^{\prime}}>0\left(v^{\prime} \in V(K)\right), \mu>0$ and $\sigma>0$ such that, as $n \rightarrow \infty$ and uniform for all $\ell \geq 0$,

$$
\operatorname{Pr}\left\{X_{n}=v\right\}=\frac{\tau_{v}}{\sqrt{n}} \exp \left(-\frac{(\ell-\mu n)^{2}}{2 \sigma^{2} n}\right)+O\left(\frac{1}{n}\right) \quad\left(v \in V\left(K_{\ell}\right)\right),
$$

where $\tilde{v}\left(\right.$ for $\left.v \in K_{\ell}\right)$ denotes the corresponding node in $K$.

Theorem 2 is, of course, a direct generalization of Theorem 1. As above the second and the third case can be generalized to functional limit theorems in the following sense. For $v \in V(L)$ let $\hat{v}:=-1$ and for $v \in V\left(K_{\ell}\right)$ set $\hat{v}:=\ell$. Then $\hat{X}_{n}$ is a process on the integers $\geq-1$ and after a proper scaling $\hat{X}_{n}$ can be approximated by a reflected Brownian motion or by a Brownian motion. Note further that the matrix $\mathbf{R}$ in the fi rst part of Theorem 2 is the classical $\mathbf{R}$-matrix for positive recurrent quasi-birth-and-death processes, it is given by $\mathbf{R}=\mathbf{C} \cdot \mathbf{M}(1)$ and satisfi es the equation $\mathbf{R}=\mathbf{C}+\mathbf{R B}+\mathbf{R}^{2} \mathbf{D}$, compare with (Latouche and Ramaswami, 1999, Theorem 6.2.1).

\subsection{Proof of the Theorem}

Proof. First, let us consider the case $x_{0}>1$ and $x_{1}=1$. By assumption, $x=1$ is a regular point of $\mathbf{M}(x)$ and, thus, the function

$$
f(x)=\operatorname{det}\left(\mathbf{I}-x \mathbf{B}^{\prime}-x^{2} \mathbf{C}^{\prime} \mathbf{M}(x) \mathbf{D}^{\prime}\right)
$$

is regular at $x=1$ and satisfi es $f(1)=0$. Equivalently, 1 is an eigenvalue of the matrix $\mathbf{B}^{\prime}+\mathbf{C}^{\prime} \mathbf{M}(1) \mathbf{D}^{\prime}$. Since the matrix $\mathbf{B}^{\prime}+\mathbf{C}^{\prime} \mathbf{M}(1) \mathbf{D}^{\prime}$ is primitive irreducible, 1 is a simple eigenvalue. Consequently $x=1$ is a simple zero of $f(x)$ (and there are no further zeros on the circle $|x|=1$ ). Hence all functions of the inverse matrix $\left(\mathbf{I}-x \mathbf{B}^{\prime}-x^{2} \mathbf{C}^{\prime} \mathbf{M}(x) \mathbf{D}^{\prime}\right)^{-1}$ have a simple pole at $x=1$ (and no other singularities on the circle $|x|=1)$. Thus, it follows as in the proof of Lemma 3 that the limits

$$
\lim _{n \rightarrow \infty} \operatorname{Pr}\left\{X_{n}=w\right\}
$$

exist for $w \in V(L)$. Similarly we get the existence of the corresponding limits for $v \in K_{\ell}$ and (12) with $\mathbf{R}=\mathbf{C M}(1)$. Since $\sum_{v \in V(G)} p_{v}=1$ the moduli of all eigenvalues of $\mathbf{R}$ have to be smaller than 1 .

Next, suppose that $x_{0}=x_{1}=1$. Now $\mathbf{M}(x)$ is singular at $x=1$ and behaves like (11). We also have $f(1)=0$ (with $f(x)$ from above) and by using the defi nition of the determinant it also follows that $f(x)$ has a square-root singularity of the form

$$
f(x)=c \sqrt{1-x}+O(1-x),
$$

where $c \neq 0$. (If we consider $s=\sqrt{1-x}$ as a new variable then it follows as in the first part of the proof that $f(x)=\tilde{f}(s)$ has a simple zero in $s$. Thus, $c \neq 0$.) 
Next, consider the powers $(x \mathbf{C M}(x))^{\ell}$. By assumption $x \mathbf{C M}(x)$ has just positive entries (for real $x$ with $0<x \leq 1)$. Hence, there exists a unique positive eigenvalue $\lambda(x)$ of $x \mathbf{C M}(x)$ such that the moduli of all other eigenvalues are smaller than $\lambda(x)$. By continuity this is also true in a neighborhood of the real axis. Thus,

$$
(x \mathbf{C M}(x))^{\ell}=\lambda(x)^{\ell} \mathbf{Q}+O\left(\lambda(x)^{(1-\eta) \ell}\right)
$$

for some matrix $\mathbf{Q}$ and some $\eta>0$. Since $\mathbf{M}(x)$ has a square-root singularity at $x=1$, the eigenvalue $\lambda(x)$ has the same property:

$$
\lambda(x)=c_{1}-c_{2} \sqrt{1-x}+O(1-x) .
$$

Hence, we are in a similar situation as in Lemma 4 and (13) and (14) follow with the only difference that an additional factor $c_{1}^{\ell}=\lambda(1)^{\ell}$ appears. However, if $c_{1}<1$ then the probabilities do not sum up to 1 but the sum is bounded by $O(1 / \sqrt{n})$. On the other hand, if $c_{1}>1$ then the sum of the probabilities does not converge. This provides $c_{1}=1$ and completes the proof of the second part of Theorem 2 .

Finally, suppose that $x_{1}>1$. Then we also have $x_{0}>1$. Thus, if we consider $\mathbf{M}_{\ell}(x)$ in a neighborhood of $x=1$ then all components of $\mathbf{M}_{\ell}(x)$ behave (almost) as powers of $\lambda(x)$ (the largest eigenvalue of $x \mathbf{C M}(x)$ ) that is now analytic at $x=1$. Thus, we can again use the (saddle point) methods of Drmota (1994) and obtain (15), however, again with a factor $\lambda(1)^{\ell}$. As above it follows that $\lambda(1)=1$ and we are done. Note that $\mu=1 / \lambda^{\prime}(1)$.

\section{Acknowledgements}

The author wants to thank Helmut Prodinger for pointing out the problem of bin-packing and Wolfgang Woess for many useful hints and references. He is also grateful to three anonymous referees for their careful reading, for their constructive comments, and for further references.

\section{References}

M. Drmota. A bivariate asymptotic expansion of coeffi cients of powers of generating functions. Europ. J. Combinatorics, 15:139-152, 1994.

M. Drmota. Systems of functional equations. Random Struc. Alg., 10:103-124, 1997.

M. Drmota, D. Gardy, and B. Gittenberger. A unifi ed presentation of some urn models. Algorithmica, 29: 120-147, 2001.

M. Drmota and B. Gittenberger. On the profi le of random trees. Random Struc. Alg., 10:421-451, 1997.

W. Feller. An introduction to probability theory and its applications. Vol. I. Third edition. John Wiley \& Sons Inc., New York, 1968.

W. Feller. An introduction to probability theory and its applications. Vol. II. Second edition. John Wiley \& Sons Inc., New York, 1971.

Y. Guivarc'h. Application d'un théorème limite local à la transience et à la récurrence de marches de Markov. In Théorie du potentiel (Orsay, 1983), volume 1096 of Lecture Notes in Math., pages 301332. Springer, Berlin, 1984. 
H. Hermanns, U. Herzog, and J.-P. Katoen. Process algebra for performance evaluation. Theor. Comput. Sci., 274(1-2):43-87, 2002.

A. Krámli and D. Szász. Random walks with internal degrees of freedom. II. First-hitting probabilities. Z. Wahrsch. Verw. Gebiete, 68(1):53-64, 1984.

W. Kuich and F. J. Urbanek. Infi nite linear systems and one-counter languages. Theoret. Comput. Sci., 22 (1-2):95-126, 1983.

S. P. Lalley. Return probabilities for random walks on the half line. J. Theoretical Probability, 8:571-599, 1995.

S. P. Lalley. Random walks on regular languages and algebraic systems of generating functions. In Algebraic methods in statistics and probability (Notre Dame, IN, 2000), volume 287 of Contemp. Math., pages 201-230. Amer. Math. Soc., Providence, RI, 2001.

S. P. Lalley. Random walks on infi nite free products and infi nite algebraic systems of generating functions. manuscript, 2002.

G. Latouche and V. Ramaswami. Introduction to matrix analytic methods in stochastic modeling. ASASIAM Series on Statistics and Applied Probability. Society for Industrial and Applied Mathematics (SIAM), Philadelphia, PA, 1999.

M. F. Neuts. Matrix-geometric solutions in stochastic models, volume 2 of Johns Hopkins Series in the Mathematical Sciences. Johns Hopkins University Press, Baltimore, Md., 1981. An algorithmic approach.

M. F. Neuts. Structured stochastic matrices of $M / G / 1$ type and their applications, volume 5 of Probability: Pure and Applied. Marcel Dekker Inc., New York, 1989.

H. Prodinger. Some remarks on a paper: "On the mean behaviour of on-line packing algorithms" by W. Knödel. Elektron. Informationsverarb. Kybernet., 21(1-2):3-7, 1985.

H. Prodinger. Further results on a problem of Knödel concerning the analysis of bin-packing. In Numbertheoretic analysis (Vienna, 1988-89), volume 1452 of Lecture Notes in Math., pages 193-198. Springer, Berlin, 1990.

W. Woess. Random Walks on Infinite Graphs and Groups. Cambridge University Press, Cambridge, 2000. 